\title{
Pharmacokinetic Concentration Subcategory
}

National Cancer Institute

\section{Source}

National Cancer Institute. Pharmacokinetic Concentration Subcategory. NCI Thesaurus.

Code C87971.

A subdivision of pharmacokinetic concentration data. 